\title{
DRUG RESISTANCE AND ACANTHAMOEBA KERATITIS: THE QUEST FOR ALTERNATIVE ANTIPROTOZOAL CHEMOTHERAPY
}

\author{
JOHN HAY ${ }^{1}$, COLIN M. KIRKNESS ${ }^{1}$, DAVID V. SEAL ${ }^{1}$ and PETER WRIGHT ${ }^{2}$ \\ Glasgow' and London
}

\begin{abstract}
SUMMARY
Trophozoites and cysts of 20 isolates of Acanthamoeba from the cornea and five from related samples were tested in vitro for sensitivity to ten drugs (three aromatic diamidines, two aminoglycosides, two macrolides, a polyene macrolide antibiotic, an organoarsenical and an antimetabolite) and two cationic antiseptics (chlorhexidine and polyhexamethylene biguanide, PHMB). Only chlorhexidine and PHMB showed uniform amoebacidal activity. Aromatic diamidines (pentamidine isethionate, propamidine isethionate and diminazene aceturate) generally proved effective against both forms of the amoeba; only pentamidine gave synergy with the biguanide while propamidine gave an additive effect. Other drugs tested proved erratic or ineffective against different isolates. Chlorhexidine alone, or together with propamidine, was subsequently used in two patients with proven Acanthamoeba keratitis; the causative isolates were sensitive to the individual compounds and to the combination in vitro. The treatment provided resolution of the clinical disease; amoebae were shown to be nonviable by histology and culture. The combination of chlorhexidine and propamidine is recommended for treatment of proven Acanthamoeba keratitis.
\end{abstract}

Keratitis associated with Acanthamoeba infection is a relatively rare, sight-threatening condition occurring most often in contact lens wearers, ', where there has been inappropriate or inadequate disinfection of contact lens systems. ${ }^{2}$ The clinical presentation of the disease is often mistakenly diagnosed as herpes simplex or fungal infection. ${ }^{3}$ This results in inappropriate anti-microbial agents being administered. Early features ${ }^{4-6}$ such as pain, photophobia and recurrent epithelial breakdown with little infil-

From: 'Department of Bacteriology, The Royal Infirmary, Glasgow; ${ }^{2}$ Department of Ophthalmology, Tennent Institute, University of Glasgow, Glasgow, UK.

Correspondence to: Professor C. M. Kirkness, FRCS (Edinburgh and Glasgow), FRCOphth, Department of Ophthalmology, Tennent Institute, University of Glasgow G11 6NT, UK. trate, dendritiform patterns and localised oedema, especially in a young person, as well as an association with previous contact lens wear, should suggest the possibility of Acanthamoeba keratitis. This can be confirmed by isolation and cultivation of the protozoan from corneal scrapes or biopsy material. ${ }^{7}$ Once a definitive diagnosis has been achieved, appropriate anti-acanthamoebal drug therapy will invariably be required.

The literature attests to a wide variety of drugs providing variable efficacy against different Acanthamoeba species or strains, both in vivo and in vitro. In clinical practice, however, it is important to note that some of the agents, for example some of the aromatic diamidines, may merely inhibit replication or induce encystment of the tropozoite form, ${ }^{8}$ rendering it quiescent as a cyst, and thus often resistant to conventional drug therapy. In such circumstances the cyst retains the potential to exacerbate the disease on discontinuation of the drugs or, if infected tissue is retained, following corneal transplantation. ${ }^{9}$

In the United Kingdom, empirical combination therapy of propamidine, dibromopropamidine and neomycin has proved efficacious in some patients. ${ }^{10}$ Drug resistance, ${ }^{11}$ as well as allergic or toxic reactions after prolonged therapy with propamidine, ${ }^{12}$ has limited the use of this combination, and has prevented its widespread acceptance in clinical practice.

Novel approaches to chemotherapy of Acanthamoeba keratitis continue to be forthcoming. For example, a formulation containing the cationic antiseptic polyhexamethylene biguanide at fairly low concentration, alone or in combination with propamidine and/or neomycin, has proved very effective against both trophozoites and cysts of Acanthamoeba derived from proven clinical cases of the infection. ${ }^{13.14}$ Drug therapy, however, may be complicated by a number of factors, mostly associated with failure to attend to the natural history and metabolism of the protozoan within the diseased cornea and also to the pharmacology of the selected $\operatorname{drug}(\mathrm{s})$ within this location. 
The purpose of this study was to suggest a more rational approach to chemotherapy of Acanthamoeba keratitis based on in vitro drug sensitivity studies on cultures of Acanthamoeba isolated from patients and contact lens accoutrements (details of which have been published previously). Representatives of selected drug classes and cationic antiseptics were used singly or, where considered appropriate, in combination with each other, in order to determine whether a potent acanthamoebacidal action could be identified in vitro, where the mechanism of action could be established, and the effect subsequently exploited in vivo. Illustrative case reports are used to highlight some important aspects.

\section{MATERIALS AND METHODS}

Acanthamoeba and Cultivation

Eighteen Acanthamoeba corneal isolates, including three from the same patient (TB) taken at different time intervals, were initially investigated. From one of these (AT), isolates from the soft contact lens and its storage case were also included. From another (MT), an isolate from the contact lens storage case and one from the water supply at work (the home was negative) were included.

On completion of testing these isolates, another patient (AB) presented with Acanthamoeba keratitis. Isolates from a corneal biopsy and scrape as well as a soft contact lens were examined. This afforded the opportunity of assessing the in vitro findings from the 18 samples in a clinical setting.

All amoebae were maintained by routine passage on to $1.5 \%$ high clarity bacteriological agar No. 1 (LAB M) made up in amoebal saline. ${ }^{15}$ It was spread with heat-killed Klebsiella aerogenes and moistened intermittently with amoebal saline prior to incubation in air at either $25^{\circ} \mathrm{C}$, $32{ }^{\circ} \mathrm{C}$ or $35^{\circ} \mathrm{C}$.

In order to obtain sufficient numbers of each of the 25 isolates for in vitro drug screening, the surface of each plate was flooded with amoebal saline and agitated in order to permit transfer of amoebae to sterile plastic $75 \mathrm{~cm}^{2}$ tissue-culture flasks (Sterlin, CelCult) containing approximately $50-100 \mathrm{~cm}^{3}$ of a defined growth medium. ${ }^{16}$
After several transfers, the amoebae were incubated in this medium for approximately 72 hours at either $25{ }^{\circ} \mathrm{C}$ or $32{ }^{\circ} \mathrm{C}$.

In order to determine the purity of Acanthameoba cultures, Giemsa staining was performed. Viability of each Acanthamoeba culture was assessed using a $0.2 \%$ trypan blue. Cyst populations were obtained by incubating trophozoite cultures for about 7 days at $25{ }^{\circ} \mathrm{C}$ or $32{ }^{\circ} \mathrm{C}$. Purity and viability were determined retrospectively. as described above, on excysted cohorts of amoebae. Only cultures with $>98 \%$ purity and viability were used for drug sensitivity studies.

\section{Drugs}

Aqueous solutions $(100 \mu \mathrm{g} / \mathrm{ml})$ of drug or disinfectant were prepared immediately prior to use and filter-sterilised using Gelman filters with $0.22 \mu \mathrm{m}$ pore size. Compounds used for assessment of amoebacidal action are shown in Table I. These agents were selected mainly as a consequence of literature reports of their efficacy, or that of related drugs or antiseptics, against Acanthamoeba, either in vitro or in vivo.

\section{Drug and Antiseptic Screening}

Drug and antiseptic screening was performed using a series of sterile 96-well microtitre plates containing a standardised concentration of $2 \times 10^{4}$ organisms per $100 \mu \mathrm{l}$ of medium per well. One hundred microlitres of doubling dilutions of each compound $(100-0.8 \mu \mathrm{g} / \mathrm{ml})$ were produced vertically for each of the 12 compounds tested. Lids were secured, then the contents of plates mixed gently for 10 minutes on a plate rotator prior to incubation at either $25{ }^{\circ} \mathrm{C}$ or $32{ }^{\circ} \mathrm{C}$.

Sensitivity of isolates was assessed after 48 hours of incubation, by recording either the lowest concentration of drug or antiseptic which resulted in complete lysis or degeneration of trophozoites and non-viability of resulting cysts (minimum trophozoite amoebacidal concentration, MTAC) or, for cysts, the lowest concentration of test compound that resulted in no excystment and trophozoite replication (minimum cysticidal concentration, MCC), ${ }^{13}$

Table I. Drugs and antiseptics used in this study, their classification and proposed antimicrobial mechanism of action

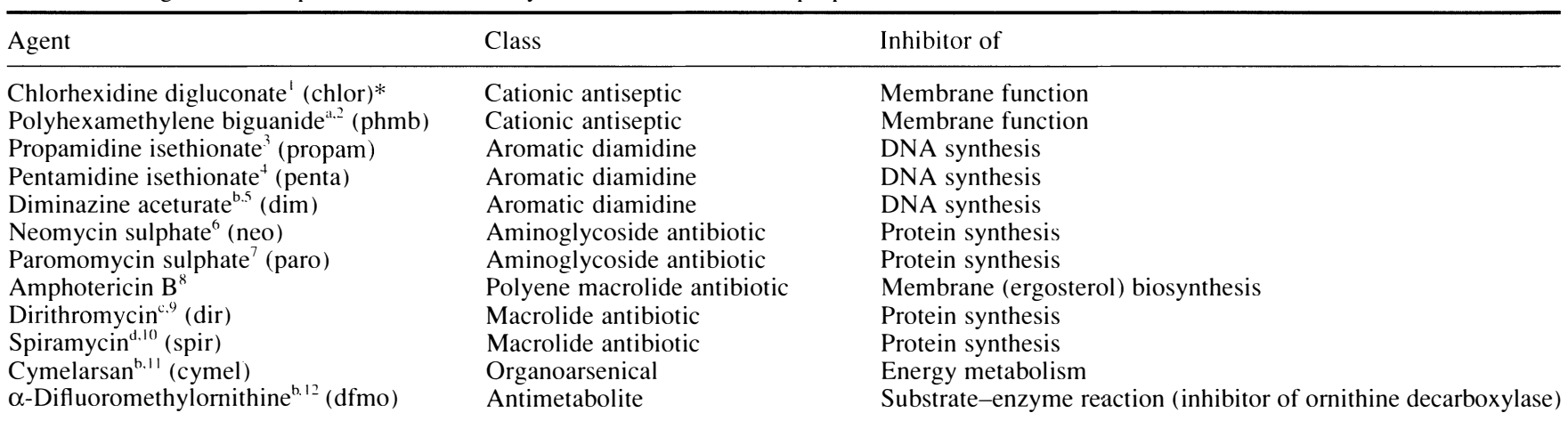

Gift from: "Moorfields Eye Hospital; 'Prof. F. W. Jennings; 'Lilly Research Centre Ltd; 'Rhone-Poulenc Ltd.

Supplied as solutions: ' $0.05 \%$ Sterets, Unisept; ${ }^{2} 0.02 \%$ contains hypromellose eye drops, $0.3 \%$; ${ }^{3} 0.1 \%$ Brolene, May \& Baker (contains benzalkonium chloride); ${ }^{6}$ Minims, Smith \& Nephew (contains phenylmercuric nitrate).

Supplied as solids: ${ }^{4}$ Pentacarinat, May \& Baker; ${ }^{7}$ Sigma; ${ }^{8}$ Fungizone i/v, Squibb: ${ }^{5.7-12}$ donated compounds.

*Abbreviations in parentheses are used in Figs. 1 and 2 and Table II. 
after thoroughly washing cysts free of residual drug, and re-incubation in the medium described above. ${ }^{16}$ Observations were performed in duplicate using an inverted microscope.

For in vitro combination testing, a chequerboard method was used. ${ }^{17}$ With this procedure, four possible outcomes of drug-drug or drug-antiseptic combinations were possible: ${ }^{18}$

1. Additivity, where the result with the two compounds was equivalent to their sum when used separately.

2. Autonomy (or indifference), where the result with the two compounds was not different from the result with the more effective compound used alone.

3. Antagonism, where the result with the two compounds was less than the additive response.

4. Synergism, where the result with the two compounds was greater than the additive response.

Five replicates per determination were performed ${ }^{19}$ using the combinations shown in Table II; findings were based on results obtained from 7 of the 18 corneal isolates.

\section{CASE REPORTS}

\section{Patient TB}

Drug resistance occurred after treatment of Acanthamoeba keratitis with propamidine alone; topical neomycin had been withdrawn because of allergy. " The isolate collected in early treatment was retested for drug and antiseptic sensitivities, as were two later isolates. Both the latter have been recorded before as temperature-sensitive and propamidine-resistant. ${ }^{11}$

\section{Patient AT}

Treatment was given with topical betamethasone-neomycin combination q.i.d. for 4 weeks, followed by propamidine q.i.d. alone for 3 weeks, prior to laboratory confirmation of Acanthamoeba keratitis by corneal scrape and biopsy. The isolated protozoan grew poorly at $35^{\circ} \mathrm{C}$, compared with those isolated from the contact lens and storage case. AT had worn Acuvue (Johnson \& Johnson) disposable soft contact lenses (FDA Group 4) and had used Softab (Alcon; a chlorine-based system) following the manufacturer's instructions for contact lens disinfection. ${ }^{20}$

AT responded satisfactorily over a 12 month period to a combination of propamidine and neomycin, before undergoing a penetrating keratoplasty due to scarring in the visual axis. Histology revealed only a few degenerate cysts. There was no recurrence of infection after 1 year.

\section{Patient MT}

Treatment of this patient's Acanthamoeba keratitis was initially with a topical propamidine-neomycin combination, which induced a toxic reaction in the cornea within 2 months. At this stage trophozoites were still present in a corneal biopsy.

Guttae chlorhexidine $(0.02 \% \mathrm{w} / \mathrm{v}$ in $0.9 \%$ saline $)$ alone was administered for 9 months without a further adverse reaction. With this course of treatment relapse of Acanthamoeba infection was not apparent. Isolates from a corneal biopsy, contact lens and its storage case grew confluently at $35{ }^{\circ} \mathrm{C}$. MT had worn Acuvue disposable soft contact lenses (FDA Group 4) and used Softab for contact lens disinfection..$^{20}$

\section{Patient $A B$}

A 48-year-old man with mild atopy and early keratoconus had recently changed contact lenses from daily-wear soft to rigid, gas-permeable lenses. Total (Allergan), an all-inone sterilising solution containing hydrogen peroxide, was used for lens hygiene. The patient attended an eye casualty department with a dendritiform corneal ulcer, stromal oedema and a mild anterior chamber reaction. This was considered to be herpetic kerato-uveitis and treatment was commenced with acyclovir ointment and prednisolone $0.5 \%$ drops. He was then referred to one of us (C.M.K.). Intensive antiviral and steroid therapy did not bring about improvement. The eye became increasingly painful, injected, and a ring abscess developed. A corneal biopsy was performed 2 months after initial presentation. Acanthamoeba was observed histologically in the corneal stroma and cultured from it. The protozoan was seen on the hydrogel contact lens and cultured from washings.

Treatment was commenced with $0.02 \%$ chlorhexidine in isotonic saline drops every hour for a week then 2-hourly, combined with $0.1 \%$ propamidine at the same frequency, and 3-hourly prednisolone $1 \%$. The eye became white and comfortable within 7 days though the patient remained photophobic. The abscess began regressing and the epithelium slowly healed. At 2 months there was a central stromal opacity and $3 \mathrm{~mm}$ overlying epithelial defect.

The patient next presented as an emergency with a 2-day history of sudden discomfort and further loss of vision. Thè cornea was found to be perforated centrally with an intumescent lens. An emergency keratoplasty, extracapsular lens extraction and posterior chamber lens

Table II. In vitro combination testing of selected drugs against seven Acanthamoeba corneal isolates

\begin{tabular}{|c|c|c|}
\hline Combination & Effect on MTAC & Effect on MCC \\
\hline \multicolumn{3}{|l|}{ Isolates $(T B / 2 ; 1,2,10,13 ; A B)$} \\
\hline phmb + pentamidine & Synergy (slight) & Synergy (slight) \\
\hline phmb + neomycin & Additivity & Additivity \\
\hline phmb + dirithromycin & Autonomy & Autonomy \\
\hline propamidine + neomycin & Additivity & Additivity \\
\hline pentamidine + neomycin & Additivity & Additivity \\
\hline$*$ diminazine + neomycin & Additivity & Additivity \\
\hline$*_{\text {diminiazine }}+$ dirithromycin & Autonomy & Autonomy \\
\hline$*$ pentamidine $+\mathrm{dfmo}$ & Autonomy & Autonomy \\
\hline$*^{*}$ cymelarsan + dfmo & Autonomy & Autonomy \\
\hline$*$ neomycin + dirithromycin & Autonomy & Antagonism \\
\hline \multicolumn{3}{|l|}{ Isolate $A B$} \\
\hline chlorhexidine + propamidine & $\begin{array}{l}\text { Additivity } \\
\text { Additivity }\end{array}$ & $\begin{array}{l}\text { Additivity } \\
\text { Synergy (slight) }\end{array}$ \\
\hline & $\begin{array}{l}\text { Additivity } \\
\text { Additivity }\end{array}$ & Additivity \\
\hline
\end{tabular}

MTAC, minimum trophozoite amoebacidal concentration; MCC, minimum cysticidal concentration. ${ }^{*}$ Not including AB. 

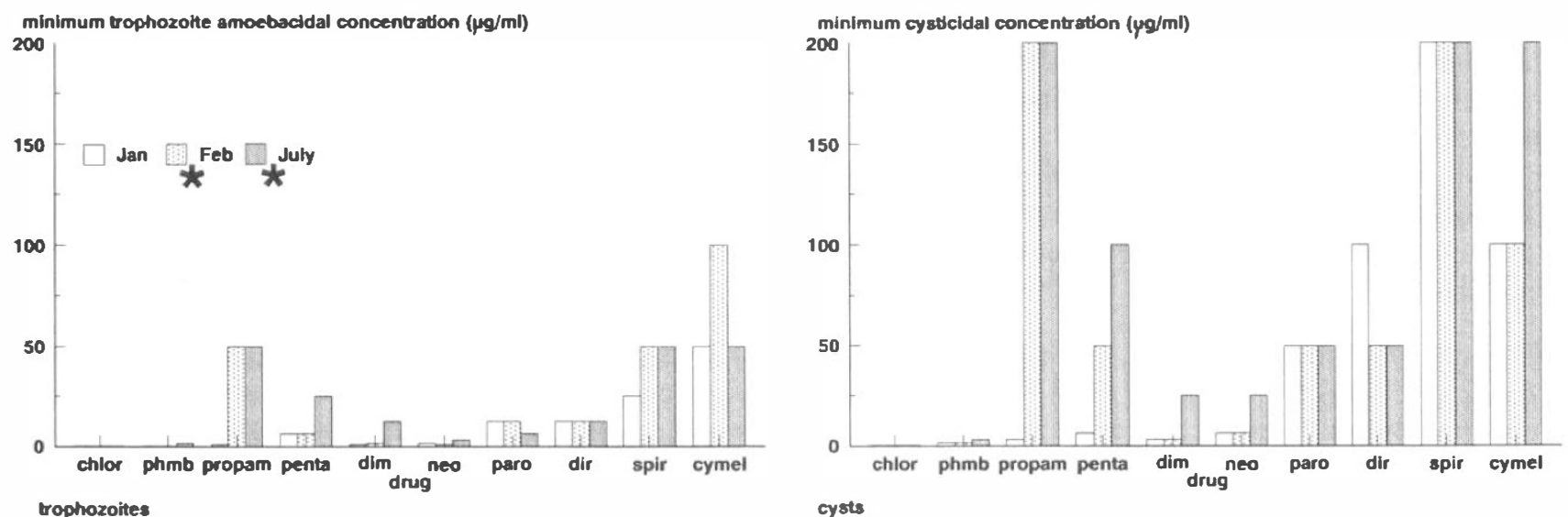

$1 \mathrm{a}$
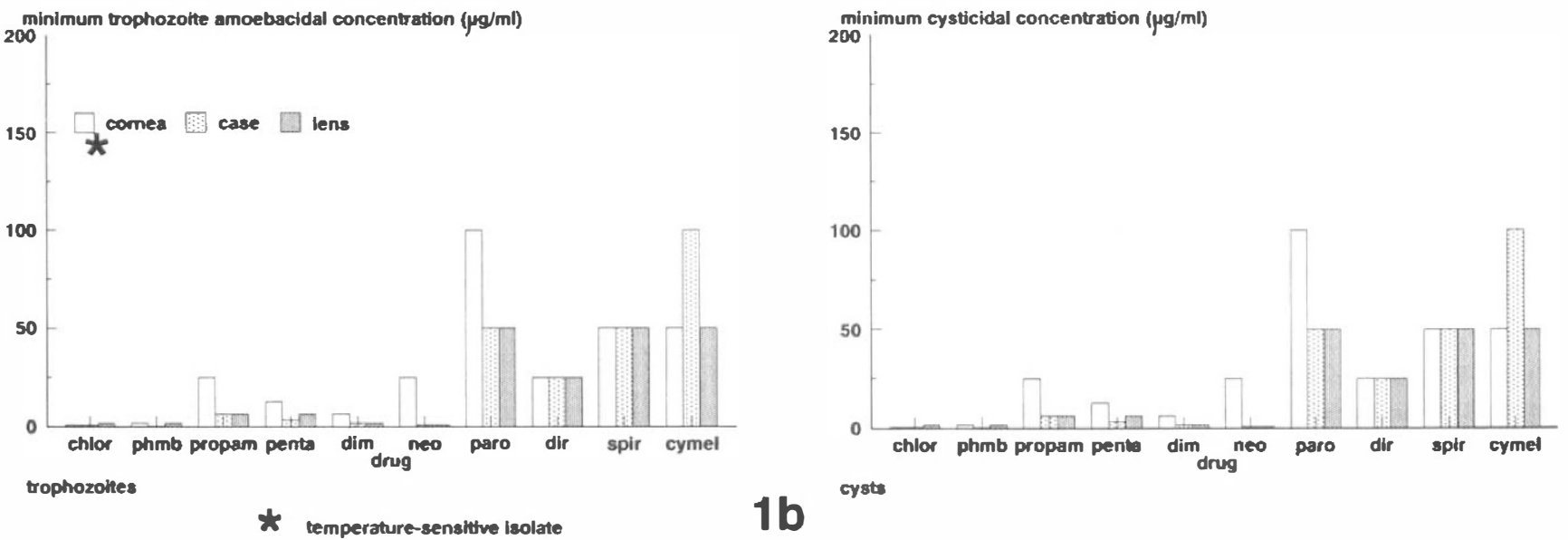

Fig. 1. (a) Minimum trophozoite amoebacidal concentration (MTAC) and minimum cysticidal concentration (MCC) often agents for three corneal isolates from patient TB. (b) MTAC and MCC of ten agents for corneal, storage case and contact lens isolates firom patient AT. For abbreviations see Table I .

implant was performed. Half the excised cornea was sent for culture and half for histopathology. Streptococcus acidominimus (weakly $\alpha$-haemolytic) was cultured from the corneal tissue but it remained persistently culturenegative for Acanthamoeba. Degenerate cysts were seen in the corneal stroma but no trophozoites. The corneal epithelium appeared healthy.

Post-operatively treatment was continued with Brolene (May \& Baker) and chlorhexidine with prednisolone (1\% 2-hourly) and Polytrim (Burroughs Wellcome) drops 2-hourly. Three months after keratoplasty the graft remained clear with no signs of further infection. The corrected vision was $6 / 12$

\section{RESULTS}

\section{Patient TB (Fig. 1A)}

Trophozoites and cysts of all three corneal isolates were highly sensitive to chlorhexidine and polyhexamethylene biguanide (PHMB). While trophozoites and cysts from the first isolate were sensitive to propamidine, the two subsequent isolates showed resistance as previously tested, a phenomenon apparently related to temperature ${ }^{11}$ but not to the action of the antiseptics. Both forms of the amoeba for all isolates were sensitive to neomycin. Amphotericin B, cymelarsan and $\alpha$-difluoromethylornithine ( $\alpha$-DFMO) were ineffective against trophozoites and cysts for all three isolates. This patient, in whom medical treatment failed with propamidine and arsenicals, ${ }^{11}$ would probably have benefited from therapy with cationic antiseptics.

\section{Patient AT (Fig. 1B)}

Trophozoites and cysts of all three isolates (cornea, contact lens and storage case) were highly sensitive to chlorhexidine and PHMB. Trophozoites and cysts from the corneal isolate, but not the others, were less sensitive to propamidine and were temperature-sensitive (confluent growth at $25^{\circ} \mathrm{C}$, poor at $35^{\circ} \mathrm{C}$ ); this trend was also evident for the other two diamidines. Neomycin was more effective than paromomycin but the corneal isolate was more resistant than the others as regards both trophozoites and cysts. Amphotericin B, cymelarsan and $\alpha$-DFMO were ineffective.

\section{Patient MT (Fig. 2A)}

Trophozoites and cysts of all three isolates (cornea, storage case, workplace water sample) were highly sensitive to chlorhexidine and PHMB and grew well at $32{ }^{\circ} \mathrm{C}$. Propamidine was more effective than either pentamidine or 

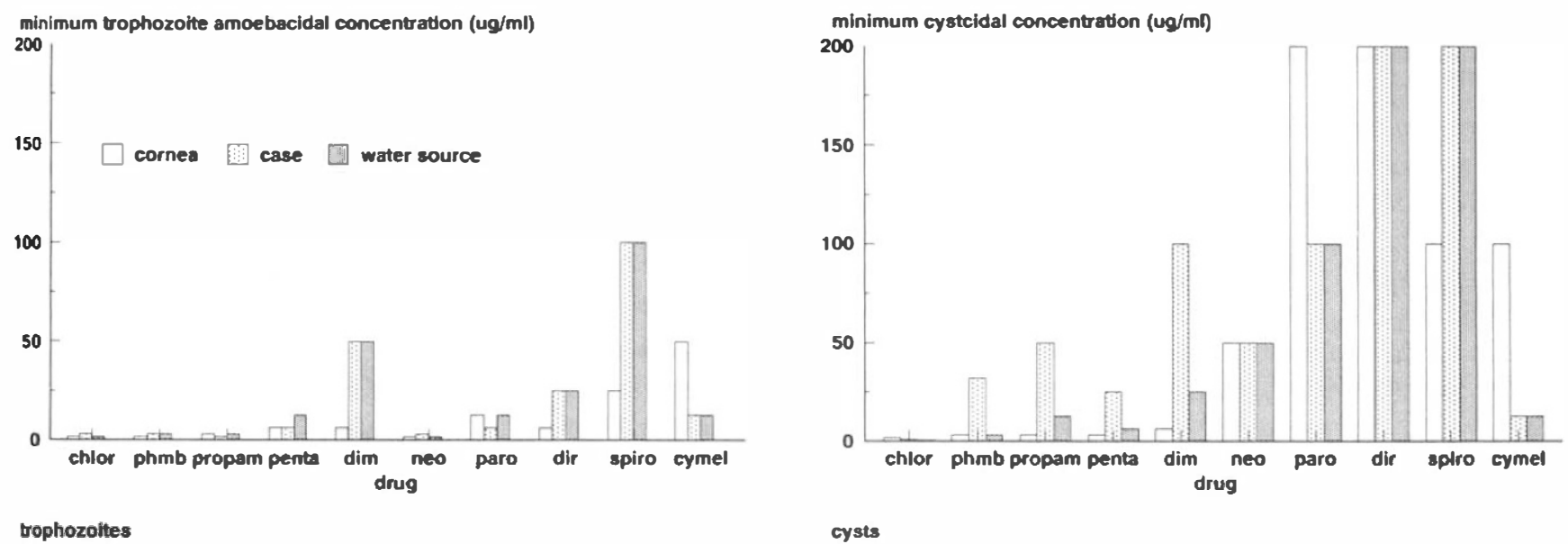

tophozoltes

2a
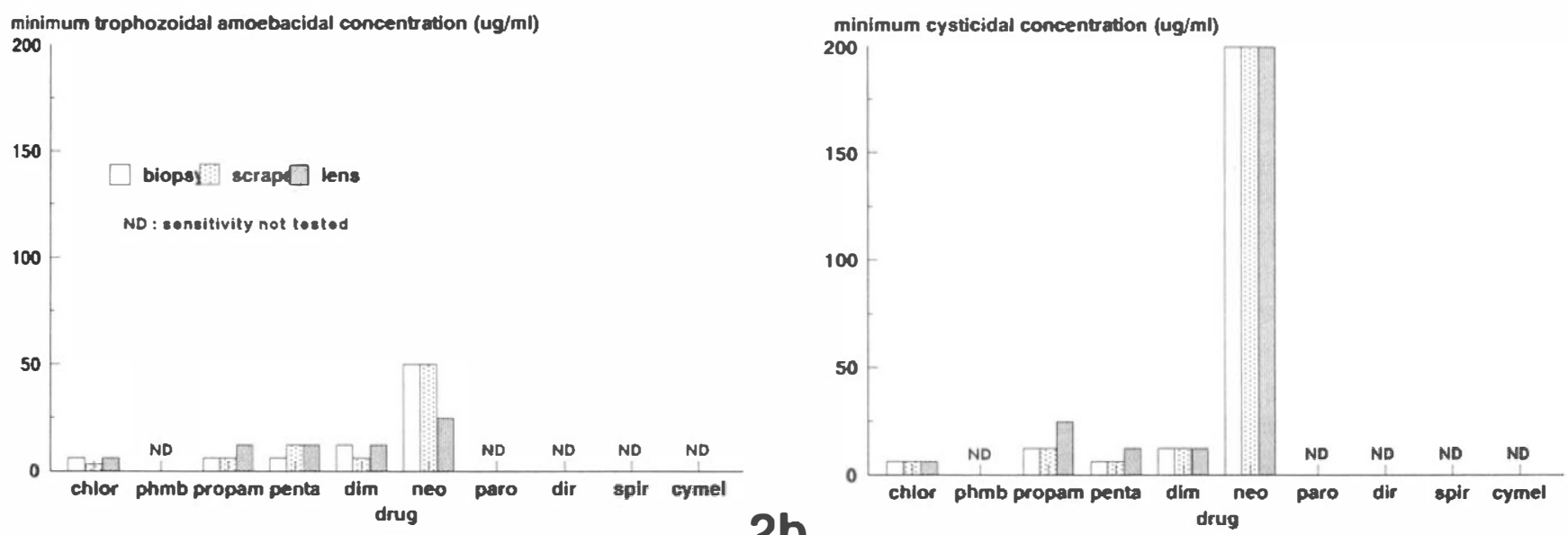

Fig. 2. (a) MTAC and MCC of ten agents for corneal, contact lens storage case and water-source isolates from patient MT. (b) MTAC and MCC of ten agents for corneal biopsy, corneal scrape and contact lens washing isolates from patient AB. For abbreviations see Table I.

diminazine against trophozoites of all three isolates, but pentamidine was most effective against cysts. For all three isolates, the storage case isolate was less sensitive to diamidines than either the corneal or water sample isolates. Trophozoites of all three isolates were sensitive to neomycin but cysts were resistant. Amphotericin B, cymelarsen and $\alpha$-DFMO were ineffective.

\section{Patient $A B$ (Fig. 2B)}

Trophozoites and cysts of all three isolates (corneal biopsy, scrape and contact lens washings) were sensitive at the upper limit to chlorhexidine; the biopsy sample only was tested against PHMB and gave similar values. All three isolates were sensitive to diamidines but again at the upper limit (MTAC 6.3-25 $\mu \mathrm{g} / \mathrm{ml}$, MCC 6.3-25 $\mu \mathrm{g} / \mathrm{ml}$ ). Trophozoites and cysts of the three isolates were resistant to acyclovir - which has been tested since it has been suggested that other antiviral agents maybe ineffective against Acanthamoeba. ${ }^{9}$

Fig. 3A-G illustrates the findings for trophozoites and cysts obtained for the remaining 13 corneal isolates. As described above, chlorhexidine and PHMB were most efficacious. All isolates with the exception of nos. 2 and 10 for trophozoites and nos. 1 and 13 for cysts were sensitive to propamidine; there were no obvious trends with the other two diamidines. Aminoglycosides were relatively ineffective against cysts while the trophozoites of four isolates (nos. 1, 4, 7, 10) were insensitive also. Trophozoites and cysts from all isolates were insensitive to macrolides, amphotericin B, cymelarsan and $\alpha$-DFMO.

Fig. $3 \mathrm{H}$ shows the average values for MTAC and MCC for 10 of the 12. drugs tested. Chlorhexidine and PHMB were the most active compounds against both trophozoites and cysts. The diamidines were the next drug class in order of acanthamoebacidal activity. Neither the aminoglycosides, macrolides nor the arsenical were effective against cysts and showed an increasing inability to destroy trophozoites.

Table II gives findings obtained from in vitro combination of selected drugs and PHMB against six corneal isolates (i.e. TB 1, 2; 1, 2, 10, 13) and selected drugs and chlorhexidine against isolate $\mathrm{AB}$. The only combinations that gave a slight synergistic response were the cationic antiseptics (chlorhexidine or PHMB) and pentamidine; 

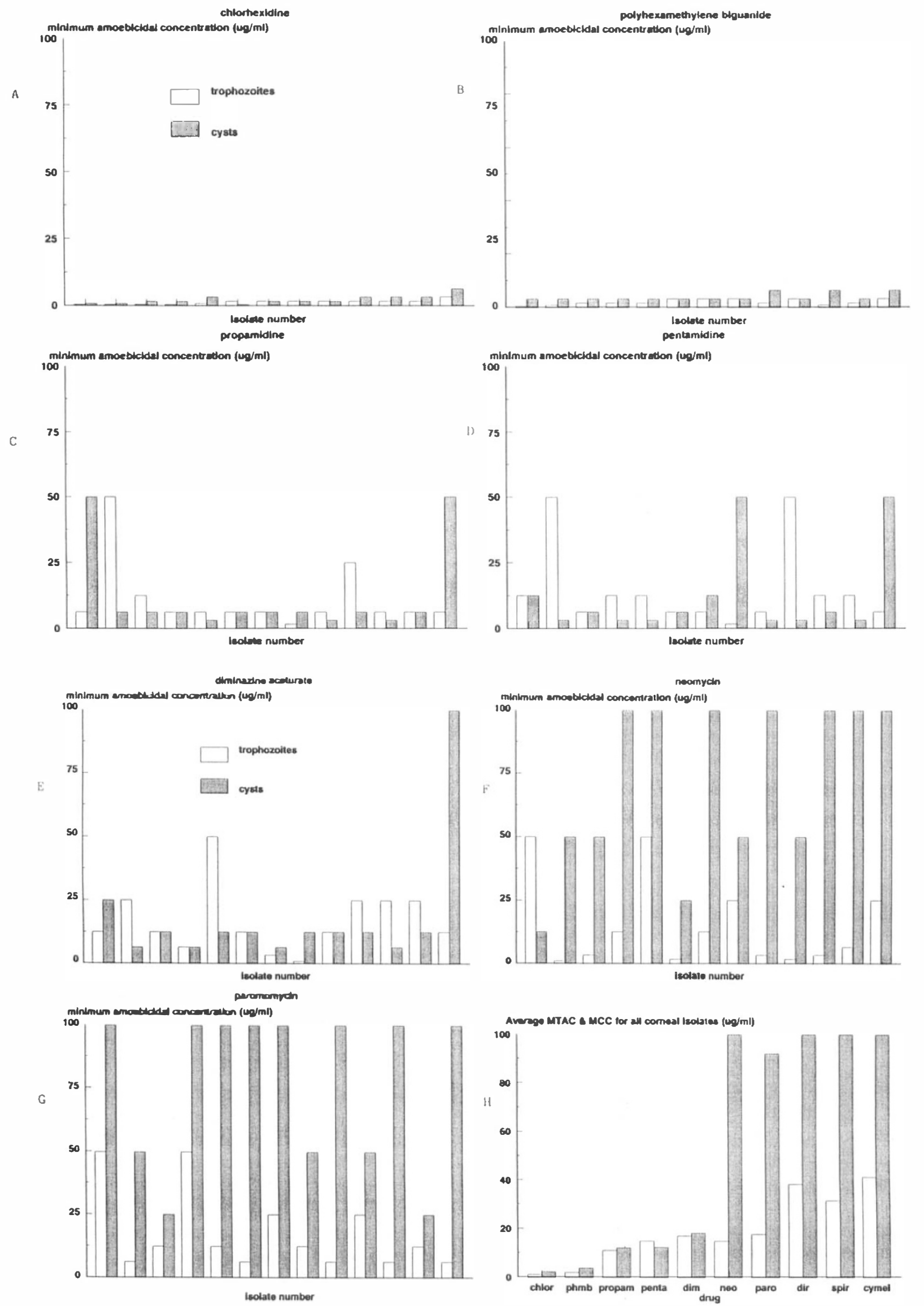

Fig. 3. MTAC and MCC for individual drugs: A, chlorhexidine; $\mathrm{B}$, PHMB; $\mathrm{C}$, propamidine; D, pentamidine; E, diminazene, $\mathrm{F}$, neomycin; $\mathrm{G}$, paramomycin; $\mathrm{H}$, average MTAC and MCC for all corneal isolates. 
additivity was found between chlorhexidine and propamidine for the $\mathrm{AB}$ isolate, a successful combination in practice. All three diamidines showed additivity with neomycin as did the combination of PHMB and neomycin. Other combinations showed autonomy, except that of neomycin and dirithromycin, where there was antagonism.

\section{DISCUSSION}

Failure of drug therapy in Acanthamoeba keratitis has been recognised for some time but the reasons are not always understood in the absence of drug sensitivity testing. This study suggests that some of the commonly used drugs such as neomycin and paromomycin are not particularly effective amoebacides - a finding in accordance with that of other workers. ${ }^{13}$ Moreover the case reports (TB, AT) suggest that resistance may develop as a result of low-dose, single-drug anti-amoebic therapy. Sensitivity testing can therefore be useful on all occasions. It is not sufficient to rely on isolation of the organism from the contact lens or its storage case since amoebae from these sources may have different sensitivities from that isolated from the cornea, particularly when the diagnosis has been late and there has been pretreatment with a variety of drugs. Since an animal model is not yet available for the sensitivity testing of Acanthamoeba isolates, in vitro assessment is a necessary but relatively unsatisfactory alternative.

Agents tested in vitro and found to have an effect on different species and strains of Acanthamoeba include: clotrimazole ${ }^{21}$ and ketoconazole, ${ }^{22}$ although like other azoles the effect is likely to be amoebastatic rather than amoebacidal, ${ }^{23}$ and with some drugs in this group the organisms may be highly resistant; ${ }^{24} 5$-fluorocytosine (a nucleotide analogue), ${ }^{25,26}$ although this drug has been found ineffective by other workers: $;^{27.28}$ the diamidines pentamidine isethionate, ${ }^{10.29}$ although others have reported insensitivity to this drug, ${ }^{22}$ hydroxystilbamidine isethionate, ${ }^{25.30}$ diminizene aceturate ${ }^{10}$ and propamidine isethionate, ${ }^{10.29}$ although again this drug has been identified as insensitive in other studies, except if combined with dimethylsulphoxide (DMSO) $;^{31}$ membrane-active peptides, the magainins,${ }^{32}$ the effect being enhanced when in combination with silver nitrate or propamidine; pimaricin; $;^{2 \times}$ amphotericin $\mathrm{B}$ or $\mathrm{AB}$ methyl ester, ${ }^{22}$ although found to be ineffective in other studies; ${ }^{27}$ certain inhibitors of folate biosynthesis $;{ }^{33}$ trifluoroperazine $;^{34}$ the aminoglycosides paramomycin, ${ }^{24.30}$ which was ineffective in other studies, ${ }^{2.5}$ and neomycin, ${ }^{30}$ also without effect in other studies except if combined with propamidine; ${ }^{31}$ polymyxin $\mathrm{E}$ (colistin); $;^{22}$ acriflavine ${ }^{30}$ (although some workers have reported resistance, $\left.{ }^{28}\right)$ and other acridines. ${ }^{35}$

There is considerable disparity regarding in vitro efficacy of drugs which are active against Acanthamoeba; none demonstrate uniform activity against all isolates, and there is differential sensitivity between trophozoites and cysts, the former being more sensitive than the latter. Furthermore, some reports are based on mixed trophozoite and cyst drug-sensitivity studies which may cause confusion with drugs that merely induce encystment and are not acanthamoebacidal in action.

Several compounds have been used with varying effect in the clinical setting. These include: itraconazole plus miconazole; ${ }^{36}$ clotrimazole; ${ }^{37}$ ketoconazole ${ }^{38}$ dibromopropamidine plus propamidine and neomycin $;{ }^{10}$ propamidine isethionate as Brolene, ${ }^{39}$ propamidine in combination with neomycin-polymyxin B-gramidicin as Neosporin (Calmic) ${ }^{40}$ Neosporin with or without miconazole or ketoconazole; ${ }^{+1}$ pimaricin plus Neodecadron (dexamethasone phosphate, neomycin sulphate) hydroxyuracil, rifampicin and atropine, $;^{28}$ PHMB solution (which contained $0.3 \%$ hypromellose, $0.45 \% \mathrm{NaCl}$, $0.37 \% \mathrm{KCl}, 0.19 \%$ borax and $0.19 \%$ boric acid) alone or in combination with propamidine,,$^{13}$ or PHMB in a solution of artificial tears combined with propamidine and neomycin. ${ }^{1+}$

As with in vitro testing, clinical reports suggest that drug selection has been relatively haphazard. As a result, it was decided to investigate a cohort of 18 corneal isolates from cases of keratitis, three from contact-lens-associated materials and one from a water supply used to clean the storage case. Most of the drugs and antiseptics used in the present study have been previously assessed for potential anti-acanthamoebal activity. Two macrolides, however, were included (dirithromycin and spiramycin), since erythromycin is known to induce encystment of Acanthamoeba.$^{42}$ The organoarsenical cymelarsen was included as a novel representative of this group with less inherent toxicity than earlier arsenical compounds which, in general, exhibit relatively poor activity against Acanthamoeba. ${ }^{11.25}$ Chlorhexidine, a cationic antiseptic, was selected for inclusion since it has been shown to have good antiacanthamoebal activity in vitro ${ }^{43}$ and had previously been suggested anecodotally for therapy by one of us (D.V.S.). PHMB, a related compound but as yet unlicenced for topical use in humans, has been shown to have considerable activity against Acanthamoeba, both in vitro and in vivo.

The cationic antiseptics showed outstanding efficacy against all isolates, with chlorhexidine giving the lowest MTAC and MCC (Fig. 3H). The aromatic diamidines as a group were second in order of efficacy, although in keeping with previous literature reports the effect varied considerably between the isolates. Interestingly, diminazine (an encystment-enhancing agent), ${ }^{8}$ showed satisfactory activity against some isolates, a finding in keeping with that of Wright et al. ${ }^{10}$ but at odds with the results of other workers. ${ }^{30}$ The aminoglycosides again showed variability amongst the strains, and had no effect against cysts, both findings confirming previous literature reports. ${ }^{13}$ Macrolides showed similar behaviour to aminoglycosides but were less effective. A note of caution should be introduced, however, since the macrolides used were prodrugs, the metabolites being more effective, at least against bacteria. ${ }^{+4}$ The organoarsenical showed poor activity against both forms of the protozoan. Others have reported similar resistance to arsenicals. ${ }^{11.25}$ In keeping with the findings of 
Table III. In vitro combination testing of drugs against Acanthamoeba corneal biopsy isolate from patient $\mathrm{AB}$

\begin{tabular}{|c|c|c|}
\hline Combination & $\begin{array}{l}\text { Effect on mean } \\
\text { trophozoite } \\
\text { amoebacidal } \\
\text { concentration }\end{array}$ & $\begin{array}{l}\text { Effect on mean } \\
\text { concentration }\end{array}$ \\
\hline phmb + pentamidine & Synergy (slight) & Synergy (slight) \\
\hline phmb + neomycin & Additivity & Additivity \\
\hline phmb + propamidine & Additivity & Additivity \\
\hline propamidine + neomycin & Additivity & Additivity \\
\hline pentamidine + neomycin & Additivity & Additivity \\
\hline chlorhexidine + propamidine & Additivity & Additivity \\
\hline chlorhexidine + pentamidine & Additivity & Synergy (slight) \\
\hline chlorhexidine + neomycin & Additivity & Additivity \\
\hline
\end{tabular}

other workers ${ }^{45}$ the inhibitor of ornithine decarboxylase, $\alpha$-DFMO, had no discernible effect on the growth of Acanthamoeba. There is, however, reason to believe that other components of polyamine metabolism in Acanthamoeba may yet be found suitable as the basis for development of more active chemotherapy against the protozoan. ${ }^{46.47}$

Single drug therapy of Acanthamoeba keratitis with currently used compounds appears inadequate, and may lead to emergence of drug resistance. Combination therapy must be considered. Notable in this context in vivo is the combination of PHMB and propamidine, ${ }^{13}$ or neomycin and propamidine; ${ }^{14}$ or neomycin, dibromopropamidine and propamidine; ${ }^{10}$ and, in vitro, DMSO and propamidine isethionate. ${ }^{31}$ In the present in vitro study, additive effects were observed with cationic antiseptics plus propamidine or neomycin, and slight synergy between the antiseptics and pentamidine (Tables II, III).

Following the demonstration of in vitro efficacy of chlorhexidine two patients have been treated with this drug. One patient (MT), who had developed an idiosyncratic reaction to both neomycin and propamidine, was treated satisfactorily with monotherapy chlorhexidine. The other patient $(\mathrm{AB})$ received combination therapy of chlorhexidine with propamidine with rapid control of the Acanthamoeba infection. It is evident, however, that useful anti-acanthamoebic drugs may not have universal activity against all amoebae. We believe that, in general, combination therapy should always be employed, firstly because of the possibility of an additive anti-amoebal effect and secondly to prevent the emergence of resistance. On the basis of these two patients, plus one other now treated successfully for 3 months with a similar combination to $\mathrm{AB}$, and on anecdotal evidence from several personal communications, chlorhexidine seems to be well tolerated in the eye.

The findings from the present study are suggestive of membrane effects, which permit easier access of drug into the amoebae. Cationic antiseptics such as chlorhexidine ${ }^{18}$ and, to a lesser extent, neomycin perturb the plasmalemma; this may facilitate the entry of an effective drug such as an aromatic diamidine. Diamidines are either inhibitors of $S$-adenosylmethionine decarboxylase in Acanthamoeba ${ }^{49}$ or drugs which interact directly with the nucleic acid of the organism. ${ }^{50.51}$ In addition they may act in a way such as occurs in human neutrophilic granulocytes by inhibition of co-factors ${ }^{52}$ or cytoplasmic enzymes,${ }^{53}$ or may on their own exert an inhibitory effect on multiplication of Acanthamoeba. Analogues of the diamidine series ${ }^{5+}$ may considerably enhance this effect.

The combination of chlorhexidine and propamidine seems to have had effective amoebacidal action within the cornea. This could shorten the time during which antiacanthamoebic drugs are required and their frequency of application. This, in turn, may reduce the likelihood of toxic reaction, ${ }^{12}$ and obviate effects of inherent ${ }^{29}$ or acquired" resistance to the diamidine.

We thank Drs D. Warhurst and J. De Jonckheere for preservation of the 'London' strains of Acanthamoeba and Mr V. Andrews, Principal Pharmacist, Moorfields Eye Hospital, for supplying the formulation of PHMB. Mrs R. Connor rendered invaluable technical assistance.

Key words: Acanthamoeba keratitis, Antiprotozoal chemotherapy, Chlorhexidine, Diamidines, Drug resistance.

\section{REFERENCES}

1. Stehr-Green JK, Bailey TM, Visvesvara GS. The epidemiology of Acanthamoeba keratitis in the United States. Am J Ophthalmol 1989;107:331-6.

2. Moore MB. Acanthamoeba keratitis and contact lens wear: the patient is at fault. Cornea 1990;9 (Suppl 1):S33-5.

3. Auran JD, Starr MB, Jakobiec FA. Acanthamoeba keratitis: a review of the literature. Cornea 1987;6:2-26.

4. Lindquist TD, Sher NA, Doughman DJ. Clinical signs and medical therapy of early Acanthamoeba keratitis. Arch Ophthalmol 1988;106:1202-6.

5. Moore MB, M Culley JP, Kaufman HE, Robin JB. Radial keratoneuritis as a presenting sign in Acanthamoeba keratitis. Ophthalmology 1986;93:1310-5.

6. Bacon AS, Dart JKG, Ficker LA, Matheson MM, Wright P. Acanthamoeba keratitis: the value of early diagnosis. Ophthalmology 1993;100:1238-43.

7. Ficker LA, Kirkness C, Wright P. Prognosis for keratoplasty in Acanthamoeba keratitis. Ophthalmology 1993;100: 105-10.

8. Byers TJ, Kim BG, King LE, Hugo ER. Molecular aspects of the cell cycle and encystment of Acanthamoeba. Rev Infect Dis 1991;13 (Suppl 5):S373-84.

9. Jones DB, Visvesvara GS, Robinson NM. Acanthamoeba polyphaga keratitis and Acanthamoeba uveitis associated with fatal meningoencephalitis. Trans Ophthalmol Soc UK 1975;95:221-32.

10. Wright P, Warhurst D, Jones BJ. Acanthamoeba keratitis successfully treated medically. Br J Ophthalmol 1985;69: $778-82$.

11. Ficker L, Seal D, Warhurst D, Wright P. Acanthamoeba keratitis: resistance to medical therapy. Eye 1990;4:835-8.

12. Johns KJ, Head WS, O’Day DM. Corneal toxicity of propamidine. Arch Ophthalmol 1988;106:68-9.

13. Larkin DFP, Kilvington S, Dart JKG. Treatment of Acanthamoeba keratitis with polyhexamethylene biguanide. Ophthalmology 1992;99:185-91.

14. Varga JH, Wolf TC, Jensen HG, Parmley VC, Rowsey JJ. Combined treatment of Acanthamoeba keratitis with propamidine, neomycin and polyhexamethylene biguanide. Am J Ophthalmol 1993;115:466-70.

15. Page FC. A new key to freshwater and soil gymnamoebae. Ambleside, Cumbria: Freshwater Biological Association, 1988.

16. Byers TJ, Akins RA, Maynard BJ, Lefken RA, Martin SM. 
Rapid growth of Acanthamoeba in defined media: induction of encystment by glucose-acetate starvation. J Protozool 1980;27:216-9.

17. Krogstad DJ, Moellering RC. Antimicrobial combinations. In: Lorian V, editor. Antibiotics in laboratory medicine, 2nd ed. Baltimore: Williams \& Wilkins, 1986:537-95.

18. King TC, Schlessinger D, Krogstad DJ. The assessment of drug combinations. Rev Infect Dis 1981;3:627-33.

19. Rand KH, Houck HJ, Brown P, Bennett D. Reproducibility of the microdilution method for antibiotic synergy. Antimicrob Agents Chemother 1993;37:613-5.

20. Seal DV, Hay J, Devonshire P, Kirkness CM. Acanthamoeba and contact lens disinfection: should chlorine be discontinued? Br J Ophthalmol 1982;77:128.

21. Stevens AR, Willaert E. Drug sensitivity and resistance of four Acanthamoeba species. Trans R Soc Trop Med Hyg 1980;74:806-8.

22. Ferrante A, Rowan-Kelly B, Thong YH. In vitro sensitivity of Acanthamoeba culbertsoni to a variety of drugs and antibiotics. Int J Parasitol 1984; 14:53-6.

23. Schuster FL. Comparative effects of selected azole compounds on trophic and cystic stages of Acanthamoeba polyphaga. J Euk Microbiol 1993;40:563-9.

24. Osato MS, Robinson NM, Wilhelmus KR. Jones DB. In vitro evaluation of antimicrobial compounds for cysticidal activity against Acanthamoeba. Rev Infect Dis 1991;13 (Suppl 5):S431-4.

25. Casemore DP. Sensitivity of Hartmanella (Acanthamoeba) to 5-fluorocytosine, hydroxystilbamidine and other substances. J Clin Pathol 1970:23:649-52.

26. Stevens AR, O'Dell WD. In vitro and in vivo activity of 5-fluorocytosine on Acanthamoeba. Antimicrob Agents Chemother 1974;6:282-9.

27. Duma RJ, Finley R. In vitro susceptibility of pathogenic Naegleria and Acanthamoeba species to a variety of therapeutic agents. Antimicrob Agents Chemother 1976;10: $370-6$.

28. Ma P, Willaert E, Jeuchter KB, Stevens AR. A case of keratitis due to Acanthamoeba in New York, New York, and features of 10 cases. J Infect Dis 1981;143:662-7.

29. Kilvington S, Larkin DFP, White DG, Beeching JR. Laboratory investigation of Acanthamoeba keratitis. J Clin Microbiol 1990;28:2722-5.

30. Nagington J, Richards JE. Chemotherapeutic compounds and Acanthamoebae from eye infections. J Clin Pathol 1976;29:648-51.

31. Saunders PPR, Proctor EM, Rollins DF, Richards JSF. Enhanced killing of Acanthamoeba cysts in vitro using dimethylsulfoxide. Ophthalmology 1992;99:1 197-200.

32. Schuster FL, Jacob LS. Effects of magainins on ameba and cyst stages of Acanthamoeba polvphaga. Antimicrob Agents Chemother 1992;36:1263-71.

33. Mehlotra RK, Shukla OP. In vitro susceptibility of Acanthamoeba culbertsoni to inhibitors of folate biosynthesis. J Euk Microbiol 1993;40:14-7.

34. Schuster FL, Mandel N. Phenothiazine compounds inhibit in vitro growth of pathogenic free-living amoebae. Antimicrob Agents Chemother 1984;25:109-12.

35. Osuna A, Rodriguez-Santiago JI, Ruiz-Perez L-M, Gamarro F, Castanys S, Giovannangeli G, et al. Antiamebic activity of new acridinic derivatives against Naegleria and Acanthamoeba species in vitro. Chemotherapy 1987;33:18-21.

36. Ishibashi Y, Matsumoto Y, Kabata T, Watanabe R, Hom- mura S, Yasuraoka K, Ishii K. Oral itraconazole and topical miconazole with debridement for Acanthamoeba keratitis. Am J Ophthalmol 1990;109:121-6.

37. Driebe WT, Stern GA, Epstein RJ, Visvesvara GS, Adi M, Komadina T. Acanthamoeba keratitis. Arch Ophthalmol 1988;106:1196-201.

38. Cohen EJ, Parlato CJ, Arentsen JJ, et al. Medical and surgical treatment of Acanthamoeba keratitis. Am J Ophthalmol 1987;103:615-25.

39. Yeoh R, Warhurst DC, Falcon MG. Acanthamoeba keratitis. Br J Ophthalmol 1987;71:500-3.

40. Moore MB, M'Culley JP. Acanthamoeba keratitis associated with contact lenses: six consecutive cases of successful management. Br J Ophthalmol 1989;73:271-5.

41. Sharma S, Srinivasan M, George C. Acanthamoeba keratitis in non-contact lens wearers. Arch Ophthalmol 1990;108: 676-8.

42. Akins RA, Byers TJ. Differentiation promoting factors induced in Acanthamoeba by inhibitors of mitochondrial macromolecule synthesis. Dev Biol 1980;78:126-40.

43. Anthony Y, Davies DJG, Meakin BJ, Halliday J, Kumar R, MacDonald I, Ritchie M. A chlorhexidine contact lens disinfection tablet: design criteria and antimicrobial efficacy in potable tap water. J Br Contact Lens Assoc 1991;14:99-108.

44. Cairns D, Hay J, Seal DV. The new macrolides: expanding the frontiers of antimicrobial chemotherapy. Pharm J 1993; 251:317-20.

45. Ferrante A, Abell TJ, Robinson B, Lederer E. Effects of singefungin and difluoromethylornithine on pathogenic free-living amoebae in vitro. FEMS Microbiol Lett 1987;40: 67-70.

46. Shukla OP, Kishore P, Gupta S, Srivastava DK. Potential metabolic targets in chemotherapy for Acanthamoeba infections: polyamine metabolism. Rev Infect Dis 1991;13 (Suppl 5):S438.

47. Shukla OP, Muller S, Walter RD. Polyamine oxidase from Acanthamoeba culbertsoni specific for $\mathrm{N}^{8}$-acetylspermidine. Mol Biochem Parasitol 1992;51:91-8.

48. Kuyyakanond T, Quesnel LB. The mechanism of action of chlorhexidine. FEMS Microbiol Lett 1992;100:211-6.

49. Hugo ER, Byers TJ. $S$-adenosyl-L-methionine decarboxylase of Acanthamoeba castellanii (Neff): purification and properties. Biochem J 1993;295:203-9.

50. Greenidge PA, Jenkins TC, Neidle S. DNA minor groove recognition properties of pentamidine and its analogs: a molecular modelling study. Mol Pharmacol 1993;43:982-8.

51. Jenkins TC, Lane AN, Neidle S, Brown DG. NMR and molecular modelling studies of the interaction of berenil and pentamidine with d(CGCAAATTTGCG) $)_{2}$. Eur J Biochem 1993:213:1175-84.

52. Arnott MA, Hay J. The effect of pentamidine salts on the NADPH-oxidase system of stimulated neutrophilic granulocytes. J Antimicrob Chemother 1990;25:247-53.

53. Arnott MA, Bennett ND, Cairns D, Hay J. Selective effects of pentamidine on cytosolic and granule-associated enzyme release from zymosan-activated human neutrophilic granulocytes. J Pharm Pharmacol 1994;46:394-6.

54. Perrine D, Barbier D, Chenu P, Georges P. Comparative study of cysticidal effects of three diamidines on Acanthamoeba strains isolated from keratitis. In: VIth International Conference on the Biology and Pathogenicity of Free-Living Amoebae 1992, abstract 288. 